Z. Wahrscheinlichkeitstheorie verw. Gebiete $55,123-132(1981)$
Zeitschrift für

Wahrscheinlichkeitstheorie und verwandte Gebiete

(C) Springer-Verlag 1981

\title{
Limit Theorems for Fourier Transforms of Functionals of Gaussian Sequences
}

\author{
M. Rosenblatt * \\ Dept. of Mathematics, University of California, San Diego \\ La Jolla, California 92093, USA
}

\begin{abstract}
Summary. Limit theorems with a non-Gaussian (in fact nonstable) limiting distribution have been obtained under suitable conditions for partial sums of instantaneous nonlinear functions of stationary Gaussian sequences with long range dependence. Analogous limit theorems are here obtained for finite Fourier transforms of instantaneous nonlinear functions of stationary Gaussian sequences with long range dependence.
\end{abstract}

\section{Introduction}

A number of authors (see [2-7]) have considered non-central limit theorems for partial sums derived from nonlinear functionals of Gaussian sequences. One considers a stationary Gaussian sequence $X_{n}, n=\ldots,-1,0,1, \ldots, E X_{n}=0, E X_{n}^{2}$ $=1$. Let the correlation function of $\left\{X_{n}\right\}$ be

$$
r(n)=E X_{0} X_{n}
$$

A real function $H(x)$ is considered with

and

$$
\int_{-\infty}^{\infty} H(x) \exp \left(-\frac{x^{2}}{2}\right) d x=0
$$

$$
\int_{-\infty}^{\infty} H(x)^{2} \exp \left(-\frac{x^{2}}{2}\right) d x<\infty
$$

The derived sequence $H\left(X_{n}\right), n=\ldots,-1,0,1, \ldots$ is defined and the limiting behavior of the sequence

$$
Y_{n}^{N}=\frac{1}{A_{N}} \sum_{j=N(n-1)}^{N n-1} H\left(X_{j}\right), \quad \begin{aligned}
& n=\ldots,-1,0,1, \ldots \\
& N=1,2, \ldots
\end{aligned}
$$

\footnotetext{
* Research supported in part by the Office of Naval Research Contract N00014-75C-0428.
} 
is determined as $N \rightarrow \infty$, where $A_{N}$ is a suitable positive norming factor. Under appropriate conditions of long-range dependence on the sequence $\{r(n)\}$ and other conditions on the function $H$, nonnormal (non-central) limiting distributions for the sequence $\left\{Y_{n}^{N}, n=\ldots,-1,0,1, \ldots\right\}$ have been obtained as $N \rightarrow \infty$.

The object of this note is to obtain analogous limit theorems for finite Fourier transforms

$$
Y_{n}^{N}(\beta)=\frac{1}{A_{N}} \sum_{j=N(n-1)}^{N n-1} H\left(X_{j}\right) e^{-i j \beta} .
$$

We could deal with the Fourier transform in complex form or equivalently the real and imaginary parts under appropriate conditions as $N \rightarrow \infty$. The behavior of the Fourier transform is of interest because it is a basic ingredient in the construction of spectral estimates [1]. Various of the ideas used in the paper of Dobrushin and Major [3] are helpful in deriving such a result.

We shall assume that the covariance

$$
r(n)=|n|^{-\alpha} L(|n|) \sum_{j=0}^{m} s_{\lambda_{j}} \cos n \lambda_{j}, \quad s_{\lambda_{j}}>0
$$

for some positive integer $m$ with $0=\lambda_{0}<\lambda_{1}<\lambda_{2}<\ldots<\lambda_{m}$ and $L(t), 0<t<\infty$, a slowly varying function, i.e.,

$$
\lim _{s \rightarrow \infty} \frac{L(s t)}{L(s)}=1
$$

for every $t \in(0, \infty)$. Let $H_{j}(x)$ be the $j$-th Hermite polynomial with leading coefficient 1. The function $H(x)$ is then expanded in terms of the Hermite polynomials

where

$$
H(x)=\sum_{j=1}^{\infty} c_{j} H_{j}(x)
$$

$$
\sum_{j=1}^{\infty} c_{j}^{2} j !<\infty
$$

For convenience, let $B_{n}^{N}$ denote

$$
B_{n}^{N}=\{j \mid j \in Z, n N \leqq j<(n+1) N\} .
$$

Also let $\Lambda_{k}$ be the set of frequencies obtained by taking sums of any $k$ elements (with repetition allowed) out of the set $\left\{0, \pm \lambda_{1}, \pm \lambda_{2}, \ldots, \pm \lambda_{m}\right\}$.

We also introduce the following complex-valued Gaussian random measures. $W_{0}$ is the spectral measure of the white noise process so that

$$
\begin{aligned}
W_{0}(\Delta) & =\overline{W_{0}(-\Delta)} \\
E\left|W_{0}(\Delta)\right|^{2} & =\frac{1}{2 \pi}|\Delta|
\end{aligned}
$$


for any interval $\Delta$. Also, for any disjoint intervals $\Delta_{1}, \ldots, \Delta_{j}$ on the positive axis $W_{0}\left(\Delta_{1}\right), \ldots, W_{0}\left(\Delta_{j}\right)$ are independent. Further $\operatorname{Re} W_{0}(\Delta), \operatorname{Im} W_{0}(\Delta)$ are independent Gaussian variables with mean zero and equal variances if $\Delta$ is an interval on the positive axis. $W_{\mu}$ for $\mu>0$ is a Gaussian random measure with the same properties as $W_{0}$ with the following exception. We no longer have (11). Also $W_{\mu}\left(\Delta_{1}\right), \ldots, W_{\mu}\left(\Delta_{j}\right)$ are independent for any disjoint intervals $\Delta_{1}, \ldots, \Delta_{j}$ on the real axis. Further the random measure $W_{-\mu}, \mu>0$, is specified so that

$$
W_{-\mu}(\Delta)=\overline{W_{\mu}(-\Delta)}
$$

for any interval $\Delta$. Such measures $W_{\mu}$ are introduced for $\mu= \pm \lambda_{1}, \ldots, \pm \lambda_{m}$ and it is assumed that $W_{\lambda_{1}}, \ldots, W_{\lambda_{m}}$ are independent.

\section{Asymptotic Distribution of Fourier Transforms}

We state our result below.

Theorem. Let (6) hold with $\alpha<\frac{1}{k}$ where $k$ is the smallest index in the series (8) for which $c_{k} \neq 0$. Set

$$
A_{N}=N^{1-\frac{k \alpha}{2}} L(N)^{\frac{k}{2}}
$$

Then the finite dimensional distributions of

tend to those of

$$
Y_{n}^{N}(\beta), \quad n=\ldots,-1,0,1, \ldots, \beta \in \Lambda_{k},
$$

given by

$$
Y_{n}^{*}(\beta), \quad n=\ldots,-1,0,1, \ldots, \beta \in \Lambda_{k},
$$

$$
\begin{aligned}
Y_{n}^{*}(\beta)= & D^{-k / 2} c_{k} \int e^{i n\left(x_{1}+\ldots+x_{k}\right)} \frac{e^{i\left(x_{1}+\ldots+x_{k}\right)}-1}{i\left(x_{1}+\ldots+x_{k}\right)} \\
& \cdot\left|x_{1}\right|^{\frac{\alpha-1}{2}} \ldots\left|x_{k}\right|^{\frac{\alpha-1}{2}} \\
& \cdot \sum_{\mu_{1}+\ldots+\mu_{k} \equiv \beta \bmod 2 \pi}^{\prime}\left(s_{\mu_{1}} \frac{1+\delta\left(\mu_{1}\right)}{2} \ldots s_{\mu_{k}} \frac{1+\delta\left(\mu_{k}\right)}{2}\right)^{\frac{1}{2}} \\
& \cdot W_{\mu_{1}}\left(d x_{1}\right) \ldots W_{\mu_{k}}\left(d x_{k}\right)
\end{aligned}
$$

where

$$
D=\int_{-\infty}^{\infty} \exp (i x)|x|^{\alpha-1} d x=2 \Gamma(\alpha) \cos \left(\frac{\alpha \pi}{2}\right)
$$

and $\Sigma^{\prime}$ denotes a sum over $k$-tuples of $\mu$ values where the $\mu_{j}$ 's can only range over $0, \pm \lambda_{1}, \ldots, \pm \lambda_{m}$.

The multiple Wiener integral has to be modified so as to take account of the fact that $W_{0}(-d x)=\overline{W_{0}(d x)}$ and $W_{-\mu}(-d x)=\overline{W_{\mu}(d x)}$. See [2] for a discussion of such questions. 
For convenience let

and

$$
\lambda_{-j}=\lambda_{j}
$$

$$
s_{\lambda_{-j}}=s_{\lambda_{j}}, \quad j=1, \ldots, m .
$$

Lemma 1. Let $a>0$ be any real number satisfying

$$
a<\frac{1}{2} \min _{i \neq j}\left|\lambda_{i}-\lambda_{j}\right|
$$

Then there is an infinitely differentiable symmetric function $f$ satisfying

$$
\begin{aligned}
0 \leqq f(x) \leqq 1 & \text { for all } x \\
f(x) \equiv 1 & \text { for all }|x|<a / 2 \\
f(x) \equiv 0 & \text { for all }|x|>a .
\end{aligned}
$$

Let

and

$$
\begin{gathered}
X_{n}=\int_{-\infty}^{\infty} e^{i n x} d z(x), X_{j}=\int_{-\infty}^{\infty} e^{i n x} f\left(x-\lambda_{j}\right) d z(x)=\int_{-\infty}^{\infty} e^{i n x} f_{j}(x) d z(x) \\
j=-m, \ldots, m
\end{gathered}
$$

$$
\begin{aligned}
{ }_{\phi} X_{n} & =X_{n}-\sum_{j=-m}^{m}{ }_{j} X_{n}=\int_{-\pi}^{\pi} e^{i n x} f_{\phi}(x) d z(x) \\
& =\int_{-\pi}^{\pi} e^{i n x}\left(1-\sum_{j=-m}^{m} f_{s}(x)\right) d z(x) .
\end{aligned}
$$

Let $r_{j}(n)$ be the covariance sequence of ${ }_{j} X_{n}, j=-m, \ldots, m, \phi$. Then

$$
\begin{aligned}
r_{j}(n) & =\int e^{i n x}\left|f_{j}(x)\right|^{2} d G(x) \\
& =\frac{1}{2}\left(1+\delta_{\lambda_{j}}\right)|n|^{-\alpha} L(|n|) s_{\lambda_{j}} e^{i n \lambda_{j}}(1+o(1))
\end{aligned}
$$

if $j=-m, \ldots, m$ and

$$
r_{\phi}(n)=\int_{-\pi}^{\pi} e^{i n x}\left|f_{\phi}(x)\right|^{2} d G(x)=o\left(|n|^{-\alpha} L(|n|)\right) .
$$

Here $G$ is the spectral measure of the stationary Gaussian sequence $\left\{X_{n}\right\}$.

It is easy to construct a function $f$ having the desired properties. Consider

$$
g(x)=\left\{\begin{array}{cl}
0 & \text { if } x \leqq 0 \text { or } x \geqq 1 \\
\exp \left\{-\frac{1}{x}-\frac{1}{1-x}\right\} & \text { if } 0<x<1
\end{array}\right.
$$

Let

$$
A=\int_{0}^{1} g(u) d u
$$

and

$$
h(x)=A^{-1} \int_{0}^{x} g(u) d u
$$


Then we can take

Consider now

$$
f(x)=h\left(\frac{x}{2 a}-2\right) h\left(2-\frac{x}{2 a}\right)
$$

with

$$
r_{j}(n)=\int_{-\infty}^{\infty} e^{i n x} h_{j}(x) d G(x)
$$

Let

Then

$$
h_{j}(x)=\left|f_{j}(x)\right|^{2} .
$$

$$
h_{j}(x)=\sum c_{j, n} e^{i n x} .
$$

$$
\begin{aligned}
\int e^{i n x} h_{j}(x) d G(x)= & \sum_{k} c_{j, k} r(k+n) \\
= & \sum_{p=-m}^{m} \frac{1}{2} s_{\lambda_{p}}\left(1+\delta_{\lambda_{p}}\right) \sum_{k} c_{j, k}|n+k|^{-\alpha} L(|n+k|) \\
& \cdot e^{i(n+k) \lambda_{p}} .
\end{aligned}
$$

However if $j=-m, \ldots, m$

$$
\begin{aligned}
& \sum_{k} c_{j, k} e^{i k \lambda_{j}}=h_{j}\left(\lambda_{j}\right)=\left|f_{j}\left(\lambda_{j}\right)\right|^{2}=1 \\
& \sum_{k} c_{j, k} e^{i k \lambda_{p}}=h_{j}\left(\lambda_{p}\right)=\left|f_{j}\left(\lambda_{p}\right)\right|^{2}=0
\end{aligned}
$$

Since $h_{j}$ is infinitely differentiable

$$
c_{j, k}=O\left(|k|^{-\beta}\right)
$$

where $\beta>0$ can be chosen arbitrarily large. Thus for $j=-m, \ldots, m$

$$
\begin{aligned}
\sum_{k} c_{j, k}|n+k|^{-\alpha} L(|n+k|) e^{i(n+k) \lambda_{j}} \\
=\sum_{|k|<\sqrt{n}}+O\left(n^{-2 \alpha}\right) \\
=|n|^{-\alpha} L(|n|) e^{i n \lambda_{j}}\left(\sum_{|k|<\sqrt{ } n} c_{j, k} e^{i k \lambda_{j}}\right)(1+o(1)) \\
=|n|^{-\alpha} L(|n|)(1+o(1))
\end{aligned}
$$

and by a similar argument

$$
\sum_{k} c_{j, k}|n+k|^{-\alpha} L(|n+k|) e^{i(n+k) \lambda_{p}}=o\left(|n|^{-\alpha} L(|n|)\right)
$$

if $p \neq j$. In the same way, one can show that

$$
\sum c_{\phi, k}|n+k|^{-\alpha} L(|n+k|) e^{i(n+k) \lambda_{p}}=o\left(|n|^{-\alpha} L(|n|)\right)
$$

for all $p=-m, \ldots, m$. The lemma follows from these observations. 
Proof of the Theorem. Let $I$ denote the index set $\{-m, \ldots, 0, \ldots, m, \phi\}$. Then

$$
X_{n}=\int_{-\pi}^{\pi} e^{i n x} d Z_{G}(x)=\sum_{j \in I}{ }_{j} X_{n}
$$

where $G$ is the spectral measure of the Gaussian sequence $\left\{X_{n}\right\}$ and $Z_{G}$ is the corresponding random spectral measure of the process. Also

with

$$
X_{n}=\int_{-\pi}^{\pi} e^{i n x} d Z_{G_{j}}(x)
$$

and

$$
d Z_{G_{j}}(x)=f_{j}(x) d Z_{G}(x)
$$

$$
G_{j}(\lambda)=\int_{-\pi}^{\pi}\left|f_{j}(u)\right|^{2} d G(u) .
$$

Let us consider the case $H(x)=H_{k}(x)$. Now

Then

$$
\begin{aligned}
H_{k}\left(X_{n}\right) & =H_{k}\left(\sum_{j \in I} X_{n}\right)=\int e^{i n\left(x_{1}+\ldots+x_{k}\right)} Z_{G}\left(d x_{1}\right) \ldots Z_{G}\left(d x_{k}\right) \\
& =\sum_{j_{1}, \ldots, j_{k} \in I} \int e^{i n\left(x_{1}+\ldots+x_{k}\right)} Z_{G_{j_{1}}}\left(d x_{1}\right) \ldots Z_{G_{j_{k}}}\left(d x_{k}\right) .
\end{aligned}
$$

where

$$
\begin{aligned}
Y_{n}^{N}(\beta)= & \frac{1}{A_{N}} \sum_{j=N(n-1)}^{N n-1} H_{k}\left(X_{j}\right) e^{-i j \beta} \\
= & \sum_{j_{1}, \ldots, j_{k} \in I} \frac{1}{A_{N}} \int e^{i N(n-1)\left(x_{1}+\ldots+x_{k}-\beta\right)} \\
& \cdot k_{N}\left(x_{1}+\ldots+x_{k}-\beta\right) Z_{G_{j_{1}}}\left(d x_{1}\right) \ldots Z_{G_{j_{k}}}\left(d x_{k}\right)
\end{aligned}
$$

$$
\begin{aligned}
k_{N}\left(x_{1}+\ldots+x_{k}\right) & =\sum_{j \in B_{0}^{N}} e^{i j\left(x_{1}+\ldots+x_{k}\right)} \\
& =\frac{e^{i N\left(x_{1}+\ldots+x_{k}\right)}-1}{e^{i\left(x_{1}+\ldots+x_{k}\right)}-1}
\end{aligned}
$$

We wish to first show that the variance of a term of (27) with one of the subscripts $j_{i}=\phi$ or else with $j_{1}, \ldots, j_{k} \in\{-m, \ldots, m\}$ but $\lambda_{j_{1}}+\ldots+\lambda_{j_{k}} \neq \beta \bmod 2 \pi$ tends to zero as $N \rightarrow \infty$. For simplicity in notation the computation is carried out for distinct $j_{1}, \ldots, j_{k}$. The variance is then

$$
\begin{aligned}
& \frac{1}{A_{N}^{2}} \int\left|k_{N}\left(x_{1}+\ldots+x_{k}-\beta\right)\right|^{2} d G_{j_{1}}\left(x_{1}\right) \ldots d G_{j_{k}}\left(x_{k}\right) \\
& \quad=\frac{1}{N^{2-k \alpha} L(N)^{k}} \sum_{p \in B_{0}^{N}} \sum_{q \in B_{0}^{N}} r_{j_{1}}(p-q) \ldots r_{j_{k}}(p-q) e^{-i(p-q) \beta} \\
& =\frac{1}{N^{2-k \alpha} L(N)^{k}} \sum_{p \in \widetilde{B}_{N}}(N-|p|) r_{j_{1}}(p) \ldots r_{j_{k}}(p) e^{-i p \beta}
\end{aligned}
$$


where

$$
\tilde{B}_{N}=\{p \mid-N<p<N\} .
$$

If one of the subscripts $j_{i}=\phi$, the estimates (19) and (20) together with an estimation like that given in the proof of Lemma 1 in [3] imply that (28) tends to zero as $N \rightarrow \infty$.

The following lemma is helpful in showing that (28) tends to zero as $N \rightarrow \infty$ if $\lambda_{j_{1}}+\ldots+\lambda_{j_{k}} \neq \beta \bmod 2 \pi$.

Lemma 2. Let $\alpha>0$. If $L(t)$ is a slowly varying function there exist $L_{1}(t)$ and $L_{2}(t)$ such that $L(t)=L_{1}(t)+L_{2}(t)$

and

$$
L_{2}(t)=o\left(L_{1}(t)\right)
$$

is monotone decreasing.

$$
n^{-\alpha} L_{1}(n)
$$

This lemma follows from Karamata's theorem (for Karamata's theorem refer to the book of Ibragimov and Linnik).

By Lemma 2 we can estimate (28) when $\lambda_{j_{1}}+\ldots+\lambda_{j_{k}} \neq \beta \bmod 2 \pi$ by

$$
\frac{1}{N^{2-k \alpha} L(N)^{k}} \sum_{p} p^{-k \alpha} L_{1}(p) e^{i p\left(\lambda_{J_{1}}+\ldots+\lambda_{j_{k}}-\beta\right)}
$$

where $L_{1}$ is a slowly varying function such that $n^{-k \alpha} L_{1}(n)$ is monotone decreasing. The infinite sum in (31) is convergent since $\lambda_{j_{1}}+\ldots+\lambda_{j_{k}} \neq \beta \bmod 2 \pi$ and so the whole expression (31) tends to zero as $N \rightarrow \infty$. The case in which several subscripts are the same can be carried out similarly but in a more tedious manner. All these terms can therefore be neglected as $N \rightarrow \infty$. We now have to consider the terms for which $\lambda_{j_{1}}+\ldots+\lambda_{j_{k}} \equiv \beta \bmod 2 \pi$. The asymptotic behavior of one such term will be determined as $N \rightarrow \infty$ but the argument given when trivially elaborated can be applied to the linear combination of any finite number of such terms. Thus the joint asymptotic distribution of the terms can be determined as $N \rightarrow \infty$. Let us consider the term

$$
\frac{1}{A_{N}} \int e^{i N(n-1)\left(x_{1}+\ldots+x_{k}-\beta\right)} k_{N}\left(x_{1}+\ldots+x_{k}-\beta\right) Z_{G_{j_{1}}}\left(d x_{1}\right) \ldots Z_{G_{j_{k}}}\left(d x_{k}\right)
$$

of the sum (27) with $\lambda_{j_{1}}+\ldots+\lambda_{j_{k}} \equiv \beta \bmod 2 \pi$. Let

and

$$
Z_{N, j}(A)=\frac{N^{\alpha / 2}}{L(N)^{\frac{1}{2}}} Z_{G_{j}}\left(\lambda_{j}+N^{-1} A\right)
$$

$$
G_{N, j}(A)=\frac{N^{\alpha}}{L(N)} G_{j}\left(\lambda_{j}+N^{-1} A\right)
$$

with $A$ a Borel set. Our object is to show that (32) converges in distribution to that of

$$
\begin{aligned}
& D^{-k / 2} \int e^{i(n-1)\left(x_{1}+\ldots+x_{k}\right)} \frac{e^{i\left(x_{1}+\ldots+x_{k}\right)}-1}{i\left(x_{1}+\ldots+x_{k}\right)} \\
& \cdot s_{\lambda_{1}} \frac{1+\delta\left(\lambda_{1}\right)}{2} \ldots s_{\lambda_{k}} \frac{1+\delta\left(\lambda_{k}\right)}{2} W_{\lambda_{1}}\left(d x_{1}\right) \ldots W_{\lambda_{k}}\left(d x_{k}\right) .
\end{aligned}
$$


Of course, the finite dimensional distributions of (5) will converge in distribution to those of (14) and as remarked earlier a simple but notationally tedious elaboration of our argument will yield that result in the case $H(x)=H_{k}(x)$.

Let

$$
K_{N}(u)=\frac{1}{N} k_{N}\left(\frac{1}{N} u\right) .
$$

Then expression (32) can be rewritten as

$$
\int e^{i(n-1)\left(x_{1}+\ldots+x_{k}\right)} K_{N}\left(x_{1}+\ldots+x_{k}\right) Z_{N, j_{1}}\left(d x_{1}\right) \ldots Z_{N, j_{k}}\left(d x_{k}\right) .
$$

The measure $G_{N, j}(\cdot)$ corresponding to $Z_{N, j}(\cdot)$ can be shown to converge locally weakly as $N \rightarrow \infty$ to

$$
s_{\lambda_{j}}\left(\frac{1+\delta\left(\lambda_{j}\right)}{2}\right)_{0} G(\cdot)
$$

where ${ }_{0} G$ has density $D^{-1}|x|^{\alpha-1}$. Set

$$
\begin{aligned}
& \varphi_{N . \bar{\lambda}}\left(t_{1}, \ldots, t_{k}\right) \\
& \quad=\int e^{i \frac{1}{N}\left(u_{1} x_{1}+\ldots+u_{k} x_{k}\right)}\left|K_{N}\left(x_{1}+\ldots+x_{k}\right)\right|^{2} G_{N, j_{1}}\left(d x_{1}\right) \ldots G_{N, j_{k}}\left(d x_{k}\right)
\end{aligned}
$$

where $u_{p}=\left[t_{p} N\right], p=1, \ldots, k$ and $\bar{\lambda}=\left(\lambda_{j_{1}}, \ldots, \lambda_{j_{k}}\right)$. Then

$$
\begin{aligned}
\varphi_{N, \bar{\lambda}}( & \left.t_{1}, \ldots, t_{k}\right) \\
= & \frac{1}{N^{2-k \alpha} L(N)^{k}} \sum_{p \in B_{0}^{N}} \sum_{q \in B_{0}^{N}} r\left(p-q+u_{1}\right) \ldots r\left(p-q+u_{k}\right) \\
& \cdot e^{-i(p-q) \beta} e^{-i\left(u_{1} \lambda_{j_{1}}+\ldots+u_{k} \lambda_{j_{k}}\right)} \\
= & \frac{1}{N^{2-k \alpha} L(N)^{k}} \sum_{p \in \bar{B}^{N}}(N-|p|) r\left(p+u_{1}\right) \ldots \\
& \cdot r\left(p+u_{k}\right) e^{-i p \beta} e^{-i\left(u_{1} \lambda_{j_{1}}+\ldots+u_{k} \lambda_{j_{k}}\right) .}
\end{aligned}
$$

Just as in the proof of Lemma 1 of [3] one can show that

$$
\begin{aligned}
\lim _{N \rightarrow \infty} \varphi_{N, \bar{\lambda}}\left(t_{1}, \ldots, t_{k}\right) & \\
= & \int_{-1}^{1}(1-|x|) \frac{1}{\left|x_{1}+t_{1}\right|^{\alpha}} \cdots \frac{1}{\left|x+t_{k}\right|^{\alpha}} d x \\
& \cdot s_{\lambda_{j_{1}}} \ldots s_{\lambda_{j_{k}}} \frac{1+\delta\left(\lambda_{j_{1}}\right)}{2} \ldots \frac{1+\delta\left(\lambda_{j_{k}}\right)}{2} \\
= & \varphi_{\bar{\lambda}}\left(t_{1}, \ldots, t_{k}\right) .
\end{aligned}
$$

with the limit function $\varphi_{\bar{\lambda}}$ a continuous function. Also the function $\varphi_{N, \bar{\lambda}}$ of (38) is the Fourier transform $\left(u=\left(u_{1}, \ldots, u_{k}\right), u_{p}=\left[t_{p} N\right], x=\left(x_{1}, \ldots, x_{k}\right)\right)$

$$
\varphi_{N, \bar{\lambda}}(t)=\int e^{i u \cdot x / N} \eta_{N, \bar{\lambda}}(d x)
$$

of a finite measure $\eta_{N, \bar{\lambda}}$ on $R^{k}$ with support on $[-N \pi, N \pi]^{k}$. Since $\varphi_{N, \bar{\lambda}}(t)$ tends 
to a limit function $\varphi_{\bar{\lambda}}$ that is continuous, it follows that the sequence of measures $\eta_{N, \bar{\lambda}}$ tends to a finite measure $\eta_{\bar{\lambda}}$ and $\varphi_{\bar{\lambda}}$ is the Fourier transform of $\eta_{\bar{\lambda}}$ (see Lemma 2 of [3]).

We consider special functions $h$ taking on a finite number of values of the following character. Consider sets $A_{1}, A_{2}, \ldots, A_{s}, s=1,2, \ldots$, Borel sets (of finite ${ }_{0} G$ mass) such that $A_{-i}=-A_{i}$ and $A_{-s}, \ldots, A_{-1}, A_{1}, \ldots, A_{s}$ are disjoint. Let $\hat{h}\left(i_{1}, \ldots, i_{k}\right)$ be complex numbers and

$$
h\left(x_{1}, \ldots, x_{k}\right)=\widehat{h}\left(i_{1}, \ldots, i_{k}\right) \quad \text { if } x_{1} \in A_{i_{1}}, \ldots, x_{k} \in A_{i_{k}}
$$

where $i_{1}, \ldots, i_{k}$ take on the values $\pm 1, \ldots, \pm s$ but with $i_{j} \neq i_{j^{\prime}}$ if $j \neq j^{\prime}$ and $h\left(x_{1}, \ldots, x_{k}\right)=0$ for all other $x_{1}, \ldots, x_{k}$. One can show that

$$
\int h\left(x_{1}, \ldots, x_{k}\right) Z_{N, j_{1}}\left(d x_{1}\right) \ldots Z_{N, j_{k}}\left(d x_{k}\right)
$$

asymptotically as $N \rightarrow \infty$ has the same distribution as

$$
\int h\left(x_{1}, \ldots, x_{k}\right) W_{\lambda_{j_{1}}}\left(d x_{1}\right) \ldots W_{\lambda_{j_{k}}}\left(d x_{k}\right)
$$

The integral (40) is a polynomial in the random variables $Z_{N, j_{i}}(B)$ with the $B$ 's Borel sets. Now the joint distribution of the random variables $Z_{N, j_{i}}(B)$ tends to the joint distribution of the random variables $W_{\lambda_{j_{i}}}(B)$ and so we have the desired limit behavior. Also $K_{N}\left(x_{1}+\ldots+x_{k}\right)$ tends to

$$
K_{0}\left(x_{1}+\ldots+x_{k}\right)=\frac{e^{i\left(x_{1}+\ldots+x_{k}\right)}-1}{i\left(x_{1}+\ldots+x_{k}\right)}
$$

uniformly on every finite rectangle in $R^{k}$. Equations (37) and (39) imply that

$$
\lim _{A \rightarrow \infty} \int_{R^{k}-[-A, A]^{k}}\left|K_{N}\left(x_{1}+\ldots+x_{k}\right)\right|^{2} G_{N, j_{1}}\left(d x_{1}\right) \ldots G_{N, j_{k}}\left(d x_{k}\right)=0
$$

uniformly for $N=0,1,2, \ldots$. An adaptation of Lemma 3 of [3] then implies that expression (36) tends in distribution to expression (35) as $N \rightarrow \infty$. For relation (41) implies that for any $\varepsilon>0$ one can find a function of type $h$ (depending on $\varepsilon$ ) such that

$$
\begin{gathered}
E \mid \int\left[e^{i(n-1)\left(x_{1}+\ldots+x_{k}\right)} K_{N}\left(x_{1}+\ldots+x_{k}\right)-h\left(x_{1}, \ldots, x_{k}\right)\right] \\
\left.\cdot Z_{N, j_{1}}\left(d x_{1}\right) \ldots Z_{N, j_{k}}\left(d x_{k}\right)\right|^{2}<\varepsilon
\end{gathered}
$$

for $N>N(\varepsilon)$ and

$$
\begin{gathered}
E \mid \int\left[e^{i(n-1)\left(x_{1}+\ldots+x_{k}\right)} K_{N}\left(x_{1}+\ldots+x_{k}\right)-h\left(x_{1}, \ldots, x_{k}\right)\right] \\
\left.\cdot W_{\lambda_{j_{1}}}\left(d x_{1}\right) \ldots W_{\lambda_{j_{k}}}\left(d x_{k}\right)\right|^{2}<\varepsilon .
\end{gathered}
$$

The same argument as that given on p. 36 of [3] implies the validity of the theorem for general $H$.

The theorem was derived for stationary sequences. However, it is clear that a corresponding result could have been obtained for random fields under conditions comparable to those given in [3]. The normalization in the theorem is of the form $N^{\gamma} L(N)$ with $\gamma>\frac{1}{2}$ and $L(N)$ slowly varying. The greatest interest is most likely in the case of a non-Gaussian limiting process. However, one can obtain nonnormal limiting distributions with a normalization having exponent 
$\gamma<\frac{1}{2}$ by considering processes somewhat like those analyzed in [6] and [7]. A parallel argument carried out in the case of continuous time parameter suggests that the limiting distributions obtained are self-similar.

\section{Additional Remarks}

Taqqu noted orally that one can determine non-Gaussian self-similar processes whose second order properties are the same as the Wiener process by using the techniques of [6] or [7]. This means that the exponent in the self-similarity is $1 / 2$ as it is for the Wiener process.

In all of the results discussed in [3] and [4] there is a smallest index in a Hermite expansion that plays a basic role. Given the results of [4] it is easy and of interest to concoct special examples in which one needs much more than one index to characterize a self-similar distribution. Such examples are particular cases of a general class of such processes. Let ${ }_{k} W, k=1,2, \ldots$, be independent processes each having the same probability structure as $W_{0}$. Let

$$
\begin{aligned}
U_{n}(k)= & \int \exp \left[i n\left(x_{1}+\ldots+x_{k}\right)\right] K_{0}\left(x_{1}, \ldots, x_{k}\right) \\
& \cdot\left|x_{1}+\ldots+x_{k}\right|^{\frac{1}{4}}\left|x_{1}\right|^{3 /(4 k)-\frac{1}{2}} \ldots\left|x_{k}\right|^{3 /(4 k)-\frac{1}{2}} \\
& \cdot{ }_{k} W\left(d x_{1}\right) \ldots{ }_{k} W\left(d x_{k}\right) .
\end{aligned}
$$

The discussion in [4] indicates that each of the process $\left\{U_{n}(k), n=\ldots\right.$, $-1,0,1, \ldots\}, k=1,2, \ldots$ is self-similar with exponent $1 / 2$. Let

$$
U_{n}=\sum_{k=1}^{\infty} U_{n}(k) g_{k}
$$

where the $g_{k}$ are assumed to approach zero sufficiently fast as $k \rightarrow \infty$ so that (43) converges in mean square. The resulting process $\left\{U_{n}\right\}$ is still self-similar with exponent $1 / 2$ but involves polynomial forms in the $W$ processes of all powers.

Acknowledgement. I wish to thank a referee who suggested using Lemmas 1 and 2 .

\section{References}

1. Brillinger, D.: Time series: data analysis and theory. New York: Holt, Rinehart and Winston 1975

2. Dobrushin, R.L.: Gaussian and their subordinated self-similar random generalized fields. Ann. Probab. 7, 1-28 (1979)

3. Dobrushin, R.L., Major, P.: Non-central limit theorems for nonlinear functionals of Gaussian fields. Z. Wahrscheinlichkeitstheorie verw. Gebiete 50, 27-52 (1979)

4. Major, P.: Limit theorems for nonlinear functionals of Gaussian sequences. [To be published in Z. Wahrscheinlichkeitstheorie verw. Gebiete]

5. Rosenblatt, M.: Independence and dependence, Proc. 4th Sympos. Math. Statist. Probab. 431443. Univ. California (1961)

6. Rosenblatt, M.: Some limit theorems for partial sums of quadratic forms in stationary Gaussian variables. Z. Wahrscheinlichkeitstheorie verw. Gebiete, 49, 125-132 (1979)

7. Taqqu, M.S.: Weak convergence to fractional Brownian motion and to the Rosenblatt process. Z. Wahrscheinlichkeitstheorie verw. Gebiete 31, 287-302 (1975)

Received March 6, 1980; in revised form September 20, 1980 